\title{
The Young Innovators of Cellular and Molecular Bioengineering
}

To highlight "the best and brightest" young faculty working in the area of cellular and molecular bioengineering, the Cellular and Molecular Bioengineering journal created a special issue featuring the "Young Innovators" within the field. The field is continuing to grow and, as such, the number of new faculty working in this developing area continues to expand. This Young Innovators issue was designed to profile the best research being carried out by the most talented assistant professors working in the area of cellular and molecular bioengineering.

In mid 2013, advertisements were created and widely distributed to solicit self-nominations for the Young Innovator special issue. Self-nominations were an important part of the process to encourage young investigators to promote themselves rather than asking for a more established investigator to help in the nomination process. The only criteria of the competition were that applicants must be current BMES members in good standing, and at the rank of assistant professor in a tenure track position or the equivalent. As part of the self-nomination process, each investigator submitted a biosketch and abstract. These were then reviewed and scored, and the most meritorious applications were selected based on the track record of accomplishments of the applicant and the quality and importance of the abstract. After being selected, the applicant was invited to submit a full-length manuscript that underwent a rigorous peer review process following the guidelines of the journal.

Within this inaugural special issue, the 13 investigators and their research teams have published their most cutting edge work and have set the bar high for next year's competition of the 2015 CMBE Young Innovators. Overall, the quality is exceptionally strong and represents some of the most exciting work currently being pursued in the field of Cellular and Molecular Bioengineering. Articles from a diverse array of research under the umbrella of "Cellular and Molecular Bioengineering" are featured, including work from areas such as systems biology, drug delivery, cell mechanics and biomaterials. Unique to this issue of the journal, each article is accompanied by a short biography of the corresponding author.

In this issue, Jan Lammerding and colleagues use a novel microfluidic platform to investigate the role of nuclear deformability in $3 \mathrm{D}$ migration. Importantly, they demonstrate that nuclear translation is a rate-limiting step, indicating that nuclear mechanics, mediated by lamins, is a critical component to cell invasion and migration. Also in the area of mechanics, Allen Liu et al. demonstrate a novel mechanism by which mechanosensitive ion channels can be opened through integrin-cytoskeletal linked forces. While these channels are present in bacteria, there is the potential to express them in mammalian cells for use in drug delivery and transport applications.

Several Young Innovators have developed novel drug delivery systems or drug testing platforms that have the potential to change how drugs are designed, fabricated or tested. Kathryn Miller-Jensen's laboratory contributed a paper on the efficacy of co-drugging in anti-HIV therapy, providing evidence that decisions on whether to co-drug are patient-specific and can be optimized quantitatively to achieve robust treatment and minimize side effects. These results underscore the importance of personalized medicine in HIV treatment. Junghae Suh and her lab describe a novel approach to increasing the efficiency of gene delivery via engineered, MMP-activatable viruses. Since MMPs are overexpressed in a number of diseases, including cancer and atherosclerosis, these agents could find broad clinical use. A novel drug testing platform is described by Hossein Tavana and colleagues using a biphasic mixing platform as a means for high throughput fabrication of tumor spheroids. Such a platform could greatly streamline chemotherapeutic drug screening.

Novel materials to control cell function is another core theme of the work of multiple Young Innovators. Adam Feinberg's laboratory describes a new "shrink wrapping" methodology in this issue that can be used to envelope cells in a defined extracellular matrix. Their approach offers a new way to recreate the complexities found in the extracellular matrix in vivo. Thomas Gaborski's lab uses novel nano-porous substrates to demonstrate that co-culture of adipose-derived stem cells (ADSCs) with endothelial cells (ECs) can induce the differentiation of ADSC into EC and induce ADSC to adopt features of pericytes. The use of these novel substrates offers the opportunity to study co-cultures that are in very close $(\mathrm{nm})$ proximity without cell-cell contact, mimicking the in vivo microenvironment of numerous tissues. Krishanu Saha and colleagues explore the effects of nanofibrous substrates on the reprogramming of fibroblasts into 
iPSCs, a key step in developing scaffolds which direct cell differentiation. Also in this issue, Ankur Singh's lab describes the fabrication of novel materials that allow for the control of both cell-adhesive chemistry and substrate mechanics. These materials enable the encapsulation of cells within an array format where mechanics and ligand availability can be independently tuned, providing a functional platform for screening.

In several exciting papers, cellular engineering approaches have revealed interesting insights into disease progression. Pamela Kreeger's lab has shown that extracellular matrix type is critical to the development of an in vitro model of endometriosis. Given our limited understanding of endometriosis, limited treatment options, and the small (but growing) community of engineers working in this field, Kreeger's paper represents a critical step in establishing a basis for engineering approaches to probe mechanisms underlying endometriosis progression. Keith Neeves and colleagues used a microfluidic vascular injury model and a nitric oxide-releasing polymer to investigate the role of nitric oxide signaling in platelet aggregation. His unique approach enabled the description of the relative roles of soluble guanyl cyclase dependent and independent pathways, an important finding to the control of clotting. In work investigating cardiomyocytes, Lauren Black's group contributed a paper demonstrating that depolarization can help maintain cell proliferation in culture. These findings could have a significant impact on cardiac tissue engineering for pediatric patients, where limitations in cardiomyocyte proliferation can hinder the development of engineered cardiac tissue. David Merryman's laboratory used a combination of experiment and modeling to dissect the $\alpha$ SMA pathways that lead to myofibroblast differentiation from fibroblasts. These results point to specific intracellular targets to control fibroblast differentiation, a key component of fibrosis.
The Young Innovators will present these findings at the 2014 Annual Biomedical Engineering Society Meeting in San Antonio, TX, in October. There will be two sessions dedicated to the Young Innovators, and we encourage you to attend and learn more about the work ongoing in their labs. It will be a wonderful opportunity to hear some of the best work being done by the rising stars of cellular and molecular bioengineering.

The 2015 Young Innovators competition is now accepting self-nominations until November 15, 2014. Interested BMES members who hold a tenure track position at the rank of Assistant Professor (or equivalent) should submit a 200-word abstract and a 2-page NIH-style biosketch to Editor-in-Chief Michael King, at mike.king@cornell.edu. Please consider nominating yourself or passing along the information to your eligible colleagues. We look forward to introducing the 2015 Class of Young Innovators at the 2015 Annual BMES Meeting in Tampa, Florida!

\section{Cynthia A. Reinhart-King}

Department of Biomedical Engineering Cornell University, 302 Weill Hall, 526 Campus Rd, Ithaca, NY 14853, USA

Electronic mail: cak57@cornell.edu

\section{DAVID J. MOONEY}

School of Engineering and Applied Sciences and Wyss Institute for Biologically Inspired Engineering, Harvard University, Cambridge, MA 01238, USA

\section{DAVID V. SCHAFFER}

Departments of Bioengineering and Chemical Engineering, California Institute for Quantitative Biosciences, and Helen Wills Neuroscience Institute, University of California, Berkeley, CA 94720, USA 